\title{
20 Jahre Welttuberkulosetag - (K)ein Grund zum Feiern
}

Ein kritischer Blick des Stop-TB Forums

DOI 10.17886/EPIBULL-2016-018

Seit zwei Dekaden wird der 24. März offiziell als jährlicher Welttuberkulosetag begangen." Dieses Datum ist eine wichtige Gelegenheit zur kritischen Reflektion - auch für zivilgesellschaftliche Akteure wie das Stop-TB Forum. Bestehend aus acht Mitgliedern, setzt sich dieser Zusammenschluss deutscher Nichtregierungsorganisationen (NROs) aus den Bereichen Medizin, Entwicklungszusammenarbeit und humanitäre Hilfe seit 2008 für die weltweite Bekämpfung der Tuberkulose ein. ${ }^{* *}$

Als Advocacy-Netzwerk sensibilisiert das Stop-TB Forum deutsche PolitikerInnen und die deutsche Öffentlichkeit für die globalen Gesundheitsgefahren der Krankheit. Es fordert zum einen die intensivere Berücksichtigung von Tuberkulose im deutschen entwicklungspolitischen Kontext etwa über bessere Finanzierung von Behandlungsprogrammen in stark betroffenen Ländern sowie eine umfassende Bekämpfung der sozialen Ursachen der Erkrankung. Zudem steht die Stärkung von Forschung und Entwicklung für bessere Diagnostika, effektivere und verträglichere Tuberkulosebehandlung sowie adäquate Impfstoffe im Fokus. Die Sicherstellung eines universellen Zugangs zu Prävention, Diagnose und Behandlung und dabei auch zu medizinischen Neuerungen bildet einen weiteren Arbeitsschwerpunkt. Die Aktivitäten für diese Zwecke reichen von parlamentarischen Diskussionsrunden für politische Stakeholder und VertreterInnen der Zivilgesellschaft bis zur aktuellen Informationsarbeit für Tuberkuloseinteressierte über den Newsletter oder Facebook.

Es ist unstrittig, dass in jüngerer Vergangenheit bedeutende Erfolge im globalen Ringen mit der „weißen Pest“ erzielt werden konnten. Besonders prominent tritt dabei das Erreichen der Vorgaben zu TB beim Entwicklungsziel 6.C der MDGs (Millennium Development Goals) der Vereinten Nationen hervor: Der bisherige Anstieg der Tuberkuloseinzidenz konnte weltweit (durchschnittlich) zum Stillstand gebracht und eine Trendumkehr bewirkt werden. ${ }^{1}$

Dennoch löste die Tuberkulose, eine der ältesten Krankheiten der Menschheit und eine behandelbare dazu, im vergangenen Jahr HIV/AIDS als weltweit tödlichste Infektionskrankheit ab. ${ }^{2}$ Zugleich steht man mit der Ausbreitung von resistenten Stämmen vor einer zunehmend dramatischen Entwicklung.

Drei Aspekte dieser Problemlage sollen hier schlaglichtartig näher beleuchtet werden.

1. Der verhaltene Optimismus angesichts zweier neu auf den Markt gekommenen Medikamente (Bedaquilin und Delamanid) darf nicht den Blick auf die klaffenden Finanzierungslücken im Bereich Forschung und Entwicklung verstellen. Wie die Treatment Action Group
(TAG) zuletzt darlegte, fehlten allein im Jahr 2014 im Tuberkuloseforschungsbereich 1,3 Milliarden US-Dollar. ${ }^{3}$ In den letzten vier Jahren zogen sich gleich drei große pharmazeutische Unternehmen aus diesem Arbeitsfeld zurück. ${ }^{4}$ Die vom Stop-TB Forum unterstützte internationale ParlamentarierInnen-Initiative des Global-TBCaucus urteilte 2014 in ihrer Barcelona Erklärung ernüchtert: „(T) he commercial market for pharmaceutical development has failed TB patients. “5

Selbst begrüßenswerte Neuerungen werfen Schwierigkeiten auf: Der Nutzen von Bedaquilin und Delamanid wird beispielsweise weiter durch eine schlechte Datenlage zur Anwendung sowie massive Zugangshürden (hoher Preis, geringe Verfügbarkeit) signifikant eingeschränkt. ${ }^{6}$ Zugleich zeigen erste nachgewiesene Resistenzen auch gegen diese zwei Präparate abermals auf, wie anpassungsfähig Mycobacterium tuberculosis ist und wie dringend daher unser Bedarf für innovative Instrumente in der Bekämpfung. ${ }^{7}$

Tuberkulose bleibt letztlich eine vernachlässigte Krankheit und verdeutlicht, angesichts Millionen Betroffener, weiter strukturelle Ungerechtigkeiten des vorherrschenden Forschungssystems.

2. Schwierig gestaltet sich auch die Finanzierung von Programmen zur Diagnose und Behandlung weltweit. Über ein Drittel der neuen Tuberkulosefälle wird laut WHO nicht diagnostiziert oder gemeldet. ${ }^{8}$ Einer der Hauptgründe ist das Fehlen von fast eineinhalb Milliarden US-Dollar jährlich. ${ }^{9}$ Neue ambitionierte Pläne, wie der Global Plan to End TB 2016-2020 mit seinem „9०-(90)-90“-Ziel, erfordern jedoch höhere finanzielle Investitionen. ${ }^{* * * *}$ Zugleich breiten sich im Osten der WHO-Region Europa Formen der resistenten Tuberkulose teils rasant aus. Ungeachtet dessen werden jedoch vielen betroffenen Staaten aufgrund ihrer Einstufung als sogenannte Länder mit mittlerem Einkommen

\footnotetext{
* Zwar betrachtete u.a. die Internationale Union gegen Tuberkulose und Lungenkrankheiten dieses Datum bereits vorher als Gedenktag, jedoch schloss sich die Weltgesundheitsorganisation (WHO) dem erst 1996 an.

**: Zu den Mitgliedern zählen Ärzte ohne Grenzen, action medeor, BUKO Pharma-Kampagne, Deutsche Lepra- und Tuberkulosehilfe (DAHW), Deutsches Zentralkomitee zur Bekämpfung der Tuberkulose, Koch-Metschnikow-Forum, Misereor und die Stiftung Weltbevölkerung. Des Weiteren ist das Stop-TB Forum Partner der TB-Europe Coalition und der Stop-TB-Partnership sowie Mitglied des Aktionsbündnisses gegen Aids.

": Das „90-(90)-90“-Ziel fordert, 90\% der weltweiten TB-Fälle zu diagnostizieren, wobei darin auch $90 \%$ der Fälle aus den am stärksten marginalisierten Gruppen enthalten sein müssen. Außerdem sollen 90\% Prozent der diagnostizierten Fälle eine erfolgreiche Behandlung zur Folge haben.
} 
internationale Gelder entzogen, darunter auch für die Bekämpfung von TB und HIV/AIDS. ${ }^{10}$

3. Elementare Fortschritte in der Tuberkulosebekämpfung wurden vor allem durch die Verbesserung der Lebensumstände erreicht. Hierzu gehört das nachhaltige Vorgehen gegen Armut und Mangelernährung sowie eine Optimierung der Wohnverhältnisse. Ebenso muss die Abkehr von Diskriminierung und Kriminalisierung von Bevölkerungsgruppen Teil der Antwort auf die internationale Herausforderung durch Tuberkulose sein.

Als weltweit viertgrößte Volkswirtschaft bleibt auch Deutschland im Bereich der Tuberkulosebekämpfung deutlich hinter den globalen Erfordernissen zurück. Die von einer breiten Koalition deutscher NROs seit Langem als überfällig eingestuften Reaktionen stehen weiterhin aus. Dies betrifft etwa das Setzen alternativer Forschungsanreize, die Einrichtung eines internationalen Forschungsfonds zur Bekämpfung vernachlässigter Krankheiten oder die Bereitstellung eines fairen Beitrags zum Globalen Fonds. So mag sich an diesem Welttuberkulosetag mancher geneigt fühlen, einem kürzlich erschienenen Beitrag der Fachzeitschrift The Lancet beizupflichten, welcher feststellte: „(I)t's time to end impotent calls for action; the demand now is for nothing less than success. "11
Literatur

1. WHO 2015: Millennium Development Goals (MDGs) Factsheet No. 290, verfügbar unter: http://www.who.int/mediacentre/factsheets/fs290/en/ (aufgerufen am 4.12.2015)

2. Stop TB Partnership 2015: The Global Plan to End TB 2016-2020, verfügbar unter http://www.stoptb.org/global/plan/plan2/ (aufgerufen am 4.12.2015)

3. TAG: 2015 Report on Tuberculosis Research Funding Trends, 2005-2014: A Decade of Data, verfügbar unter http://www.treatmentactiongroup. org/tbrd2015 (aufgerufen am 1.12.2015)

4. Treatment Action Group 2014: Tuberculosis Research at Risk as Big Pharma Shutters Programs, verfügbar unter http://www.treatmentactiongroup.org/press/tb/tbrd2014 (aufgerufen am 3.3.2016)

5. The Barcelona Declaration, verfügbar unter http://www.globaltbcaucus. org/\#!declaration/cln8o (aufgerufen am 1.12.2015)

6. MSF Access Campaign 2015: First new TB drugs in half a century reach just $2 \%$ of people who need them, verfügbar unter https://www.msfaccess.org/about-us/media-room/press-releases/first-new-tb-drugs-halfcentury-reach-just-2-people-who-need-them (aufgerufen am 3.12.2015)

7. Hoffmann et al.: Delamanid and Bedaquiline Resistance in Mycobacterium tuberculosis Ancestral Beijing Genotype Causing Extensively DrugResistant Tuberculosis in a Tibetan Refugee, in: American Journal of Respiratory and Critical Care Medicine, 2016; Vol. 193, Issue 3

8. WHO: Global Tuberculosis Report 2015, verfügbar unter http://www. who.int/tb/publications/global_report/en/ (aufgerufen am 1.12.2015)

9. The Lancet: Tuberculosis reaches new milestones, good and bad 2015, verfügbar unter http://www.thelancet.com/journals/laninf/article/ PIIS 1473-3099\%2815\%290043 1-4/fulltext (aufgerufen am 01.12.2015)

10. TB Europe Coalition: After Aid 2015: What is next for tuberculosis \& HIV in Europe?, verfügbar unter http://www.tbcoalition.eu/resources/ reports/ (aufgerufen am 4.12.2015)

11. The Lancet: Tuberculosis reaches new milestones, good and bad 2015 , verfügbar unter http://www.thelancet.com/journals/laninf/article/ PIIS1473-3099\%2815\%2900431-4/fulltext (aufgerufen am 1.12.2015)

Für diesen Beitrag danken wir Max Klein (Projektkoordinator des StopTB Forums), der auch als Ansprechpartner zur Verfügung steht (E-Mail: max.klein@stop-tb.de). 